\title{
ON A THEOREM OF HALMOS GONGERNING UNBIASED ESTIMATION OF MOMENTS
}

\author{
H. S. KONIJN \\ (received 11 August 1963)
}

\section{Introduction}

In [4] Halmos considers the following situation. Let $\mathscr{D}$ be a class of distribution functions over a given (Borel) subset $E$ of the real line, and $F$ a function over $\mathscr{D}$. He investigates which functions $F$ admit estimates that are unbiased over $\mathscr{D}$ and what are all possible such estimates for any given $F$. In particular he shows that on the basis of a sample (of size $n$ ) one can always obtain an estimate of the first moment which is unbiased in $\mathscr{D}$ and that the central moments $F_{m}$ of order $m \geqq 2$ have estimates which are unbiased in $\mathscr{D}$ if and only if $n \geqq m$, provided $\mathscr{D}$ satisfies the following properties: $F_{m}$ exists and is finite for all distributions in $\mathscr{D}$ and $\mathscr{D}$ includes all distributions which assign probability one to a finite number of points of $E$. Halmos also finds that symmetric estimates which are unbiased on $\mathscr{D}$ are unique ${ }^{1}$ and have smaller variances on $\mathscr{D}$ than unsymmetric unbiased estimates.

He recognizes that his assumptions are too restrictive for most applications and mentions in particular the case where $\mathscr{D}$ is the class of all normal distributions. The present paper addresses itself to that case.

\section{Statement of results}

If $\mathscr{D}$ is the class of all nondegenerate univariate normal distributions, then, on the basis of a sample (of size $n$ ), an estimate of the first moment which is unbiased over $\mathscr{D}$ exists (and is unique when $n=1$ ); and a central moment of order $2 r \geqq 2$ has estimates which are unbiased over $\mathscr{D}$ if and only if $n \geqq 2$, and has a unique symmetric unbiased estimate when $n=2$, but not when $n>2$.

Specifically, this means the following:

Let $z_{1}, \cdots, z_{n}$ be a sample from a normal distribution with mean $\nu$ and variance $\omega^{2}>0$. Let $\bar{z}=n^{-1} \sum z_{i}, S^{2}=\sum\left(z_{i}-\bar{z}\right)^{2}$. Recall that the even

1 It will be convenient to call a function on a $k$-dimensional Euclidean space the unique function satisfying a certain property if any other function on this space satisfying the property may differ from it only on a set of $k$-dimensional Lebesgue measure zero. 
central moments $F_{2 r}$ equal $\omega^{2 r} 2^{-r}(2 r) ! / r !$ and the odd ones vanish.

(a) If $n=1, z$ is the unique unbiased estimate of $v$, and no unbiased estimate of $\bar{F}_{2 r}$ exists for $r=1,2, \cdots 2$ In [5] this seemingly uninteresting fact turns out to be the key to a quite practical question.

(b) If $n \geqq 2$,

$$
\bar{f}_{2 r}=\frac{\{(n-3) / 2\} !(2 r) !}{\{(n+2 r-3) / 2\} ! r !}(S / 2)^{2 r}
$$

is an unbiased estimate of $\bar{F}_{2 r}(r=1,2, \cdots)$, and is the unique symmetric unbiased estimate if $n=2$, but not if $n>2$. It then follows from [6] that $\bar{z}$ and $\bar{f}_{2 r}$

(c) are the unique unbiased estimates of $\nu$ and $F_{2 r}$, respectively, which depend only on the sufficient statistic $\left(\bar{z}, S^{2}\right)$ and

(d) have the smallest variance among all unbiased estimates.

Note that $\bar{z}$ and $S^{2}$ are symmetric functions of the observations. The usual symmetric estimate $\bar{f}_{2 r}^{\prime}$ for $\bar{F}_{2 r}$, which is unbiased for all distribution functions for which $F_{2 r}$ exists, is defined only when $n \geqq 2 r$. When $r=1$ it coincides with $f_{2}$, when $r=2$ it equals $[2,27.6]$

$$
\bar{f}_{4}^{\prime}=(n !)^{-1}(n-4) !\left\{n\left(n^{2}-2 n+3\right) \sum\left(z_{i}-\bar{z}\right)^{4}-3(2 n-3) S^{4}\right\} \quad(n \geqq 4) .
$$

For any family $\mathscr{D}$ as first mentioned in the introduction or mentioned in the final section $f_{2 r}^{\prime}$ is the only symmetric estimate which is unbiased for all distributions of $\mathscr{D}$. But, if for $\mathscr{D}$ we take the class of nondegenerate univariate normal distributions, our results imply that the symmetric estimate $\bar{f}_{2 r}$ is also unbiased over this class and has a smaller variance than $\bar{f}_{2 r}^{\prime}$ for $r>1$.

In the next two sections we prove the parts of (a) and (b) which are not immediate.

\section{Nonexistence of an unbiased estimate of $\bar{F}_{2 r}$ in a sample of one}

In this section denote $z_{1}$ by $z$. If $h(z)$ is an unbiased estimate of $F_{2 r}$ then

$$
\int_{-\infty}^{\infty}\left\{h(z+\nu)-z^{2 r}\right\} \exp \left(-\frac{1}{2} z^{2} \omega^{-2}\right) d z
$$

should vanish for all $\nu$ and all $\omega>0$. This integral can be written as an

\footnotetext{
2 It has been remarked that it is obvious that from a sample of one it is not possible to oltain an unbiased estimate of two independent parameters (that is, two functions $F_{1}$ and $F_{2}$ on a class of distributions such that there exists no function $g$ in the plane with $g\left\{F_{1}(D), F_{2}(D)\right\}$ $=0$ for all distributions $D$ in the class). That this is not so is easily shown by an example. Let $\theta^{2}=\nu^{2}+\omega^{2}$, where $\nu$ and $\omega^{2}$, the mean and variance, are independent parameters when, e.g, the class is the normal class. Then $\nu$ and $\theta^{2}$ are also independent parameters over that class with unbiased estimates $z_{1}$ and $z_{1}^{2}$.
} 
integral over the positive axis and then we can make the substitution $u=z^{\frac{1}{2}}$ and obtain, setting $\omega^{\prime}=\left(2 \omega^{2}\right)^{-1}$, that

$$
\int_{0}^{\infty}\left\{h\left(-u^{\frac{1}{2}}+v\right)+h\left(u^{\frac{1}{2}}+v\right)-2 u^{r}\right\} u^{-\frac{1}{2}} \exp \left(-u \omega^{\prime}\right) d u
$$

is zero for all $v$ and all $\omega^{\prime}>0$. This being a Laplace transform of $u^{-\frac{1}{2}}$ times the expression in brackets, it follows that

$$
h(-z+v)+h(z+v)-2 z^{2 r}=0
$$

for all $\nu$ and almost all positive $z$. For all $\nu$ there is a set $S_{v}$ on the positive $z$ axis such that the Lebesgue measure $l$ of the positive points $z$ not in $S_{\nu}$ is zero and such that the above equality holds on $S_{v}$. Denote $\bigcap_{k=1,2,4,5} S_{c+a k / 2}$ by $T$.

It is easily shown ${ }^{3}$ that there exists a pair of points $a$ and $\frac{1}{2} a$ in $T$. Choosing $v=a$ and $2 a$ respectively gives for $z=a$

so that

$$
h(0)+h(2 a)=2 a^{2 r}, \quad h(a)+h(3 a)=2 a^{2 r},
$$

$$
h(0)+h(a)+h(2 a)+h(3 a)=4 a^{2 r} .
$$

Choosing $v=\frac{1}{2} a$ and $2 \frac{1}{2} a$ respectively gives for $z=\frac{1}{2} a$

so that

$$
h(0)+h(a)=a^{2 r} / 2^{2 r-1}, \quad h(2 a)+h(3 a)=a^{2 r} / 2^{2 r-1},
$$

$$
h(0)+h(a)+h(2 a)+h(3 a)=a^{2 r} / 2^{2 r-2} .
$$

Since $a \neq 0$, this is a contradiction.

\section{Uniqueness of the unbiased symmetric estimate of $\bar{F}_{2 r}$ in a sample of two and nonuniqueness in a larger sample}

For $n \geqq 2$ (so that $S^{2}$ is not identically zero) the sufficiency of the statistic $\left(\bar{z}, S^{2}\right)$ and the completeness of its distribution imply that $\tilde{f}_{2 r}$ is the unique unbiased estimate of its expectation $\bar{F}_{2 r}$ among unbiased estimates depending on $\left(\bar{z}, S^{2}\right)$ only [5]. Now if $n=2,\left(\bar{z}, S^{2}\right)$ determines the set $\left\{z_{1}, z_{2}\right\}$ of observations, but not their order. Therefore $\bar{f}_{2 r}$ is also the unique unbiased estimate of $F_{2 r}$ among unbiased estimates which are symmetric in the observations.

In general, when $n>2$, for any $a \neq 0$,

Let $a^{\prime}$ be in $T$ and let $0<b<a^{\prime}$. Define the disjoint intervals $I_{\text {, from }} i a^{\prime}$ to $i\left(a^{\prime}+b\right)$ for $i=1,2$, which have $l\left(I_{i} T\right)=i b$. Denote by $p_{i}\left(I_{i} T\right)$ the set of points $x$ in $I_{i} T$ such that $2 x / j$ is in $I_{i} T ; l(p,(I, T)\}=j b$. Now let

$$
T_{2}=T p_{2}\left(I_{1} T\right), \quad T_{1}=p_{1}\left(I_{2} T_{2}\right)
$$

then. since the $T_{i}$ are subsets of $T$ of measure $i b$, there exists $a>0$ such that $\frac{1}{2} i a$ is in $T_{i}$ for $i=1$ and 2 . In fact, there exist $c$ such that, for almost all $a$ in $T, \frac{1}{2} a$ is in $T$. For brevity use $c=0$. 


$$
\bar{f}_{2 r}+a\left\{n(n+1) \sum\left(z_{i}-\bar{z}\right)^{4}-3(n-1) S^{4}\right\}
$$

will be an unbiased symmetric estimate of $F_{2 r}$ different from $f_{2 r}$, since the mean of $\sum\left(z_{i}-\bar{z}\right)^{4}$ is $3 n^{-1}(n-1)^{2} \omega^{4}$ and the mean of $S^{4}$ is $(n-1)(n+1) \omega^{4}$, and since for $n>2$ the bracket is not identically equal to zero. For example, if $n=3,1 \frac{1}{2} \sum\left(z_{i}-\bar{z}\right)^{4}$ has mean $F_{4}+3 F_{2}^{2}$ and, in the normal case, $S^{4}$ has mean $8 F_{2}^{2}$, so that $1 \frac{1}{2}\left\{\sum\left(z_{i}-\bar{z}\right)^{4}-S^{4} / 4\right\}$ and $\frac{3}{4} \sum\left(z_{i}-\bar{z}\right)^{4}$ are unbiased estimates of $F_{4}$ different from $\bar{f}_{4}=3 S^{4} / 8$.

\section{Remarks}

One could similarly discuss unbiased estimation of other functions over the class of normal distributions.

Fraser [3] adapts Halmos' argument to cases where $\mathscr{D}$ is a certain class of distributions that have a density. Some cases of this kind have been found by Lehmann and Scheffé; see [1].

The writer is much indebted to T. C. Koopmans and T. N. Srinivasan for helpful suggestions.

\section{References}

[1] Bell, C. B., Blackwell, D., and Breiman, L., On the Completeness of Order Statistics. Ann. Math. Statist., 31, (1960), 794-7.

[2] Cramér, H. Mathematical Methods of Statistics. Princeton University Press, Princeton, (1946).

[3] Fraser, D. A. S., Completeness of Order Statistics. Canad. J. of Math., 6, (1954), 42-5.

[4] Halmos, P. R., The Theory of Unbiased Estimation. Ann. Math. Statist., 17, (1946), $34-43$.

[5] Konijn, H. S., Non-Existence of Consistent Estimator Sequences and Unbiased Estimators: A Practical Example. (To appear)

[6] Lehmann, E. L., and Scheffe, H., Completeness, Similar Regions, and Unbiased Estimation. Sankhya, 10 (1950) 305-40; 15, (1955) $219-36$.

Department of Economics,

The City College,

New York,

and

Cowles Foundation. 